\title{
CHONDROGENIC DIFFERENTIATION OF INDUCED PLURIPOTENT STEM CELLS FROM OSTEOARTHRITIC CHONDROCYTES IN ALGINATE MATRIX
}

Yiyong Wei, ${ }^{1, *}$, Wen Zeng ${ }^{1}$, Rong Wan ${ }^{1}$, Jun Wang ${ }^{1}$, Qi Zhou ${ }^{1}$, Shijing Qiu ${ }^{1,2}$ and Shree Ram Singh ${ }^{3, *}$

\author{
${ }^{1}$ Department of Orthopaedics, Institute of Traumatology and Orthopaedics, Ruijin Hospital, Shanghai Jiaotong \\ University School of Medicine, Shanghai, People's Republic of China \\ ${ }^{2}$ Bone and Mineral Research Laboratory, Henry Ford Hospital, Detroit, MI, USA \\ ${ }^{3}$ Mouse Cancer Genetics Program, National Institutes of Health, National Cancer Institute at Frederick, Frederick, \\ MD, USA
}

\begin{abstract}
Induced pluripotent stem cells (iPSCs) have the potential to revolutionise cell therapy; however, it remains unclear whether iPSCs can be generated from human osteoarthritic chondrocytes (OCs) and subsequently induced to differentiate into chondrocytes. In the present study, we investigated the differentiation potential of OCs into iPSCs using defined transcription factors and explored the possibility of using these OC-derived iPSCs for chondrogenesis. Our study demonstrates that iPSCs can be generated from OCs and that these iPSCs are indistinguishable from human embryonic stem cells (hESCs). To promote chondrogenic differentiation, we used lentivirus to transduce iPSCs seeded in alginate matrix with transforming growth factor- $\beta 1$ (TGF- $\beta 1$ ) and then in vitro co-cultured these iPSCs with chondrocytes. Gene expression analysis showed that this combinational strategy promotes the differentiation of the established iPSCs into chondrocytes in alginate matrix. Increased expression of cartilage-related genes, including collagen II, aggrecan, and cartilage oligomeric matrix protein (COMP), and decreased gene expression of the degenerative cartilage marker, vascular endothelial growth factor (VEGF), were observed. The histological results revealed a dense sulphated extracellular matrix in the co-culture of TGF- $\beta 1$-transfected iPSCs with chondrocytes in alginate matrix. Additionally, in vivo chondroinductive activity was also evaluated. Histological examination revealed that more new cartilage was formed in the co-culture of TGF- $\beta 1$-transfected iPSCs with chondrocytes in alginate matrix. Taken together, our data indicate that iPSCs can be generated from OCs by defined factors and the combinational strategy results in significantly improved chondrogenesis of OC-derived iPSCs. This work adds to our understanding of potential solutions to osteoarthritic cell replacement problem.
\end{abstract}

Keywords: Chondrocytes, chondrogenesis, differentiation, co-culture.

*Address for correspondence:

Yiyong Wei

Department of Orthopaedics

Institute of Traumatology and Orthopaedics

Ruijin Hospital, Shanghai Jiaotong University School of Medicine

Shanghai, 200025, People's Republic of China

Telephone Number: 86-21-64370045-663538

E-mail: dr.yiyongwei@yahoo.com

Alternatively: Shree Ram Singh

E-mail: singhshr@mail.nih.gov
Articular cartilage (AC) is an avascular tissue composed of chondrocytes, responsible for abundant matrix synthesis and maintenance (Merceron et al., 2011). When damaged due to traumatic or pathological conditions, AC does not heal spontaneously under physiological circumstances that result in Osteoarthritis (OA). OA is the most common musculoskeletal disease in the elderly, and could become the fourth leading cause of disability by the year 2020 (Reginster, 2002; Woolf and Pfleger, 2003; Koelling et al., 2009). Current treatment strategies are restricted to short-term symptomatic relief by pharmaceutical interventions and surgical procedures (Lohmander and Roos, 2007). Thus, treatment of cartilaginous damage poses a significant clinical challenge. Recent advances in regenerative medicine suggest that using tissue engineering-based constructs to enhance OA-cartilage repair by mobilising chondrogenic cells is a promising approach for the restoration of AC structure and function (Dehne et al., 2009; Hattori and Ohgushi, 2009; Ahmed et al., 2011).

A number of strategies have been used to identify potential cell sources for AC regeneration. It is widely agreed that tissue regeneration from autologous cells can be achieved by taking advantage of the natural course of embryonic development (DeLise et al., 2000; Tuan, 2004; Ingber et al., 2006; Goepfert et al., 2010). Therefore, autologous stem cells would be an ideal choice (Yoshida and Yamanaka, 2010). Stem cells are mainly classified into two kinds of cells: somatic stem cells and pluripotent stem cells. Somatic stem cells reside in various organs and show multipotency to regenerate damaged tissues, but have limited in vitro proliferative potential (Yoshida and Yamanaka, 2010). Therefore, pluripotent stem cells may be a better source for tissue regeneration. hESCs, the first pluripotent cells isolated from embryos, have high proliferation potential and can generate differentiated progeny of all three embryonic germ layers. However, the derivation of hESCs from early embryos raises technical and ethical limitations for their use in research and the clinic (Gaspar-Maia et al., 2011). Engineered stem cells, known as iPSCs, generated from somatic cells by transduction of defined reprogramming transcription factors, typically OCT4, SOX2, KLF4, and c-MYC, open a new avenue to avoid the controversy of using hESCs (Takahashi and Yamanaka, 2006; Takahashi et al., 2007). Because iPSCs have high proliferative and differentiation capabilities similar to those of hESCs, they hold great potential for regenerative medicine (Itoh et al., 2011). 
Recent evidence indicates that OC-secreted soluble morphogens may induce chondrogenic differentiation of human mesenchymal stem cells (MSCs) in a 3-dimensional (3D) environment while preventing their hypertrophic differentiation (Aung et al., 2011). Moreover, human OCs responded similarly non-diseased passaged dedifferentiated chondrocytes by the strategy of co-culture (Ahmed et al., 2010). In addition, a recent report suggested that bone morphogenetic protein 4-expressing dedifferentiated chondrocytes recovered a chondrocytic phenotype in vitro and that some of the dedifferentiated chondrocytes in the defect sites could undergo redifferentiation and form matrix that displayed positive toluidine blue staining for glycosaminoglycans after transplantation into the joint (Lin et al., 2008). Based on above findings, we believe that OCs are likely to serve as a cell source for the generation of iPSCs to be used in reconstruction of damaged AC. In the present study, we investigate whether iPSCs can be generated from OCs by the expression of defined transcription factors and whether these OC-derived iPSCs can be induced to differentiate into chondrocytes through a combination of lentivirus-mediated TGF- $\beta 1$ transduction and co-culture of $\mathrm{AC}$ in an alginate scaffold.

\section{Materials and Methods}

\section{Tissue sources and preparation}

Normal and osteoarthritic cartilage tissue fragments (Ruijin Hospital, Shanghai, China) were collected according to an Institutional Review Board approved protocol. Osteoarthritic cartilage samples, without signs of rheumatoid involvement, were obtained from the knee joints of a patient diagnosed according to the criteria of the American College of Rheumatology (Altman et al., 1990) with OA after total knee replacement. Control normal cartilage samples were obtained from the knee joints of an amputated patient with severe injury. Tissue samples were fixed in $4 \%$ paraformaldehyde, decalcified with 10 $\%$ ethylenediaminetetraacetic acid (EDTA, pH 7.4, SigmaAldrich, St. Louis, MO, USA), and embedded in paraffin. Paraffin sections were stained with haematoxylin/eosin (H\&E) and toluidine blue.

\section{Chondrocyte isolation and culture}

Chondrocytes from osteoarthritic and healthy cartilage were harvested following digestion for $6 \mathrm{~h}$ at $37^{\circ} \mathrm{C}$ with collagenase I (152 U/mL; Invitrogen, Carlsbad, CA, USA), collagenase II (280 U/mL; Invitrogen), and dispase (15 $\mathrm{U} / \mathrm{mL}$; Invitrogen) and underwent subsequent filtration through a $40 \mu \mathrm{m}$ mesh sieve (BD Falcon, Franklin Lakes, NJ, USA) (Koelling et al., 2009). OCs were cultured in Dulbecco's modified Eagle's medium/Ham's F-12 (DMEM/F-12, Invitrogen) medium supplemented with $10 \%$ foetal bovine serum (FBS, Invitrogen). To maintain the chondrogenic phenotype, normal chondrocytes were cultured in chondrogenic medium containing high-glucose DMEM, $20 \%$ FBS, 100 nM dexamethasone (SigmaAldrich), $50 \mu \mathrm{g} / \mathrm{mL}$ ascorbate-2-phosphate (SigmaAldrich), ITS (insulin, transferrin, and selenous acid, BD), and $1 \%$ penicillin/streptomycin (Invitrogen). Cells were seeded on coverslips, fixed in $4 \%$ paraformaldehyde and stained with toluidine blue.

\section{Lentiviral production}

Lentiviral vectors were constructed and packaged, and virus titers were measured according to a previous report (Honda et al., 2010). Human OCT4, SOX2, KLF4, c-MYC and TGF- $\beta 1$ cDNAs (Shanghai Biotechnology Co., Ltd, Shanghai, China) were inserted into the $\mathrm{pENTR/D-TOPO}$ entry vector plasmid (Invitrogen). The cDNAs in $\mathrm{pENTR/}$ D-TOPO were then transferred to the pCSII-EF-MCSIRES2-Venus lentiviral vector plasmid using Gateway LR clonase (Invitrogen). Lentiviral vectors pseudotyped with the vesicular stomatitis virus G glycoprotein (VSV-G) were produced by transient transfection of three plasmids into 293T cells: the packaging plasmid (pCAG-HIVgp), the VSV-G glycoprotein- and Rev-expressing plasmid (pCMV-VSV-G-RSV-Rev), and the lentiviral vector plasmid. The culture supernatant was concentrated using ultracentrifugation, and the viral pellet was resuspended in Hanks' balanced salt solution. Vector titers were determined by infection of 293T cells with serial dilutions of the viral concentrates supplemented with $1 \mu \mathrm{g} / \mathrm{mL}$ Polybrene (Sigma-Aldrich) and followed by fluorescence-activated cell sorting analysis of the transduced cells expressing EGFP (human OCT4, SOX2, KLF4 and c-MYC). Titers were expressed as 293T-transducing units per $\mathrm{mL}$.

\section{iPSC induction and culture}

Approximately $3 \times 10^{5}$ OCs were transduced for $24 \mathrm{~h}$ with a lentiviral cocktail carrying GFP and reprogramming factors (OCT4, SOX2, KLF4, and c-MYC), and cultured for 6 days in DMEM (Invitrogen) containing $10 \%$ FBS. Then, the cells were passaged onto a feeder layer of human amniotic epithelial cells (HAmEpiC) and cultured for $24 \mathrm{~h}$ in DMEM containing $10 \%$ FBS. One day later, the medium was changed using an ESCs-supportive medium with high glucose DMEM, 15 \% FBS, $4 \mathrm{ng} / \mathrm{mL}$ basic fibroblast growth factor (R\&D, Minneapolis, MN, USA), $2 \mathrm{mM}$ L-Gin (Sigma-Aldrich), $1 \times 10^{-4} \mathrm{M}$ nonessential amino acids (Sigma-Aldrich), $1 \times 10^{-4}$ M 2-mercaptoethanol (SigmaAldrich) and $1 \%$ penicillin/streptomycin (Invitrogen). After three weeks, the human iPSC colonies were picked, dissociated with $0.05 \%$ trypsin-EDTA (Sigma-Aldrich) digestion, plated into new culture dishes and identified. All ESC-like colonies were picked by hand and clonally expanded.

\section{Differentiation of iPSCs in vitro}

In vitro differentiation of the human iPSCs was performed using the standard embryoid body (EB) differentiation method (Li et al., 2009). Human iPSCs were cultured in culture dishes, and the cells were washed with $1 \times$ phosphate-buffered saline (PBS) without $\mathrm{Ca}$ or $\mathrm{Mg}$ (Invitrogen) before being dissociated with $0.05 \%$ trypsinEDTA. The cell suspension was pipetted and spun, and then re-suspended in ESC-supportive medium. The cell suspension was added back into the old dishes and incubated for $30 \mathrm{~min}$ to reattach HAmEpiC in the dishes. After that, the cells were collected from the dishes again and seeded onto the ultra-low attachment $100 \mathrm{~mm}$ dish 
Table 1. Applied antibodies

\begin{tabular}{|c|c|c|c|}
\hline Name & Species & Dilution & Vendor \\
\hline Anti-human SSEA-1 & mouse & $1: 100$ & R\&D \\
\hline Anti-human SSEA-4 & mouse & $1: 100$ & R\&D \\
\hline Anti-human TRA-1-60 & mouse & $1: 500$ & Chemicon \\
\hline Anti-human TRA-1-81 & mouse & $1: 500$ & Chemicon \\
\hline Anti-human NANOG & goat & $1: 100$ & R\&D \\
\hline AFP & mouse & $1: 500$ & Dako \\
\hline Desmin & mouse & $1: 100$ & Neomarkers \\
\hline Nestin & mouse & $1: 100$ & Chemicon \\
\hline
\end{tabular}

( 2 x 10\% dish) (Corning, Corning, NY, USA) in DMEM supplemented with $10 \%$ FBS to form EBs. Subsequently, the EBs were plated onto Matrigel-coated slides (BD Falcon), and 3-7 days later, the cells were fixed for immunocytochemical analysis.

\section{Directed differentiation of human iPSCs into specific cell lineage in vitro}

For 2-3 days prior to treatment, the EBs were maintained in the three different differentiation media on ultra-low plates. Following this precondition step, the EBs were transferred to gelatine-coated 6-well plates, cultured in differentiation media for 14 days with the media changed every other day and subsequently processed for gene expression analysis and histology. The 6-well plates (Corning) were coated for at least $30 \mathrm{~min}$ at room temperature with 0.1 $\%$ gelatine (Sigma-Aldrich) in $\mathrm{dH}_{2} \mathrm{O}(1 \mathrm{~mL}$ per well). The differentiation protocol was performed according to a previous report (Miki et al., 2005). Standard culture media was DMEM supplemented with $10 \%$ FBS, 2 mM L-glutamine (Sigma-Aldrich), $1 \%$ nonessential amino acid (Sigma-Aldrich), $55 \mu \mathrm{M}$ 2-mercaptoethanol (SigmaAldrich), $1 \mathrm{mM}$ sodium pyruvate (Sigma-Aldrich), and $1 \%$ antibiotic-antimycotic (Invitrogen). For pancreatic differentiation (endoderm), we used the standard medium supplemented with nicotinamide $(10 \mathrm{mM})$; for cardiomyocyte differentiation (mesoderm), we used the standard media supplemented with $1 \mathrm{mM}$ ascorbic acid 2-phosphate (Sigma-Aldrich); and for neural differentiation (ectoderm), we used the standard media supplemented with $5 \times 10^{-5} \mathrm{M}$ all-trans retinoic acid (Sigma-Aldrich) and fibroblast growth factor-4 (R\&D) at $10 \mathrm{ng} / \mathrm{mL}$.

\section{Cytochemistry and immunofluorescence assay}

Alkaline phosphatase staining was performed according to the manufacturer's instructions using the Alkaline Phosphatase Detection Kit (Millipore, Billerica, MA, USA). For the immunofluorescence assay (Miki et al., 2005), undifferentiated iPSCs and their differentiated derivatives were fixed in cold acetone for $30 \mathrm{~min}$ at $4{ }^{\circ} \mathrm{C}$, then cells were rinsed with PBS three times and incubated in protein blocking agent for $20 \mathrm{~min}$. Samples were incubated with primary antibodies (Table 1) overnight at $4{ }^{\circ} \mathrm{C}$, then rinsed with PBS three times and incubated with Cy3-conjugated secondary antibodies (R\&D) for 2 $\mathrm{h}$ at room temperature. In some experiments, cells were rinsed with PBS and mounted in aqueous mounting medium containing the nuclear counterstain DAPI $(4,6$ diamidino-2-phenylindole; Sigma-Aldrich). Images were captured using an Axio Observer A1 microscope (Carl Zeiss MicroImaging, Goettingen, Germany).

\section{Teratoma formation}

Human iPSCs were injected intramuscularly into nonobese diabetic/severe combined immune deficient (NOD/ SCID) mice $\left(4 \times 10^{6}\right.$ cells per site $)$. After $4-6$ weeks, all mice $(n=3)$ developed teratomas, which were removed and processed for $\mathrm{H} \& \mathrm{E}$ staining. All animal experiments were conducted in accordance with the Guide for the Care and Use of Animals for Research Purposes and were approved by the Shanghai Jiaotong University School of Medicine Animal Care Committee.

\section{Transfection of iPSCs with lentivirus-TGF- $\beta 1$}

Human iPSCs were infected with lentivirus carrying RFP and TGF- $\beta 1$ in the presence of polybrene $(8 \mu \mathrm{g} / \mathrm{mL})$ and plated onto a feeder layer of HamEpiC for 5 days, after which red-fluorescing human iPSCs were picked and transferred onto gelatine-coated 6-well plates, where they were cultured in DMEM medium containing $20 \%$ FBS. The observed transfection rate was about $70 \%$ for iPSCs.

\section{Co-culture of transfected iPSCs with chondrocytes in alginate matrix}

The transfected iPSCs (iPSCs/TGF- $\beta 1$ ) were released from the culture substratum and resuspended at a concentration of $0.417 \times 10^{6}$ cells $/ \mathrm{mL}$. Then iPSCs/TGF- $\beta 1$ were seeded onto the alginate matrix (Invitrogen) by pipetting the iPSCs/TGF- $\beta 1$ suspension $(300 \mu \mathrm{L})$ onto the matrix. $\mathrm{iPSC}$ /TGF- $\beta 1 /$ alginate matrix (iPSCs/TGF- $\beta 1 /$ Alginate) constructs were incubated for an additional $20 \mathrm{~min}$ to allow cell attachment in vitro, and then the composites were seeded into a 24-well culture plate and cultured for $12 \mathrm{~h}$. Normal chondrocytes were digested, counted and plated in a $24-w e l l$ culture plate at a density of $1.25 \times 10^{5}$ cells and cultured in chondrogenic medium for $12 \mathrm{~h}$.

After the above procedure was performed, the Transwell insert $(0.4 \mu \mathrm{m}$ pore size, Corning) containing 
the iPSCs/TGF- $\beta 1 /$ Alginate construct was fitted into the well containing chondrocytes, which was used as the experimental group (iPSCs/TGF- $\beta 1 /$ Alginate/ chondrocytes). The co-culture was grown in chondrogenic medium for 14 days.

Additionally, the following parallel groups were cultured in chondrogenic media for 14 days: nontransfected iPSCs/alginate matrix group (iPSCs/Alginate), non-transfected iPSCs/alginate matrix/chondrocytes group (iPSCs/Alginate/ chondrocytes) and transfected iPSCs/ alginate matrix group (iPSCs/ TGF- $\beta 1 /$ Alginate) according the same procedures (a schematic is available from the author upon request).

\section{Western blotting for TGF- $\beta 1$}

To confirm the presence of TGF- $\beta 1$ in lentivirus-mediated TGF- $\beta 1$ transduced iPSCs, western blotting was performed using a polyclonal rabbit anti-TGF- $\beta 1$ antibody (ab53169, Abcam, Cambridge, UK). Protein was extracted from the non-transfected and transfected iPSCs, and the protein concentration of the extracts was assessed using a Bradford assay kit. The optical density at $595 \mathrm{~nm}$ was measured using a spectrophotometer. The protein concentration was a standard curve obtained from a serial dilution of bovine serum albumin. The cell extracts, normalised for total protein content, were resolved using polyacrylamide gel electrophoresis and electrophoretically transferred to a supported nitrocellulose membrane. Afterwards, the membranes were blocked in $5 \%$ non-fat milk in Tris-buffered saline with $0.1 \%$ Tween (TBST) for $3 \mathrm{~h}$ and incubated with the primary antibody $(1: 500)$ at $4{ }^{\circ} \mathrm{C}$ overnight. The membranes were then washed with TBST three times for $10 \mathrm{~min}$ each, followed by the addition of anti-rabbit $\operatorname{IgG}(1: 5000)$ and enhanced chemiluminescence (ECL) visualisation of the bands.

\section{Histological analysis of iPSCs-chondrogenic differentiation}

All samples were cultured for 14 days and fixed in $4 \%$ paraformaldehyde (pH 7.4, Sigma-Aldrich) overnight at room temperature, and sections were stained with toluidine blue.

\section{Analysis of mRNA expression using reverse transcription-polymerase chain reaction (RT-PCR) and quantitative real-time PCR}

Total RNA was isolated using TRIzol (Invitrogen). RNA concentrations were determined by measuring the absorbance at $260 \mathrm{~nm}$ with a spectrophotometer. Conventional RT-PCR was performed using the SuperScript One-Step RT-PCR system (Invitrogen). Quantitative real-time PCR was carried out using SYBR green qPCR Supermix UDG (Invitrogen) and analysed with the 7300 real-time PCR system (Applied Biosystems, Foster City, CA, USA). Amplification of specific genes was performed using the primers shown in Table 2.

\section{Ectopic cartilage formation}

Five-week-old BALB/c nude mice (Shanghai Jiaotong University School of Medicine) were anaesthetised using Sumianxin $(2 \mathrm{~mL} / \mathrm{kg}$, Agricultural University, Changchun, China) administered intraperitoneally. The following groups were cultured in chondrogenic media for 14 days in vitro: iPSCs/Alginate group, iPSCs/Alginate/ chondrocytes group, iPSCs/TGF- $\beta 1 /$ Alginate group and iPSCs/TGF- $\beta 1 /$ Alginate/ chondrocytes group according

Table 2. List of primers used for PCR

\begin{tabular}{|l|l|l|}
\hline Gene & Forward & Reverse \\
\hline$\beta$-actin & CGCACCACTGGCATTGTCAT & TTCTCCTTGATGTCACGCAC \\
\hline OCT4 & GAGGAGTCCCAGGACATGAA & GTGGTCTGGCTGAACACCTT \\
\hline SOX2 & GCCGAGTGGAAACTTTTGTC & GTTCATGTGCGCGTAACTGT \\
\hline REX-1 & GCGTACGCAAATTAAAGTCCAGA & CAGCATCCTAAACAGCTCGCAGAAT \\
\hline NANOG & TCCCCAAAGCTTTTG & GGCCAGTTGTTTTTCTGCC \\
\hline GAPDH & GTGTTCCTACCCCCAATGTGT & ATTGTCATACCAGGAAATGAGCTT \\
\hline Pdx-1 & TTAGACCGAAGGGGAAAACC & TTAGGGAGCCTTCCAATGTG \\
\hline PAX-6 & CCGGCAGAAGATTGTAGAGC & CTAGCCAGGTTGCGAAGAAC \\
\hline Nkx-2.2 & GAAGAACCCCTTCTACGACA & TCGCCGCTTTCGCTTCTTG \\
\hline insulin & GCCTTTGTGAACCAACACCTG & GTTGCAGTAGTTCTCCAGCTG \\
\hline glucagon & GAGGGCTTGCTCTCTCTTCA & GTGAATGTGCCCTGTGAATG \\
\hline NSE & CCCACTGATCCTTCCCGATACAT & CCGATCTGGTTGACCTTGAGCA \\
\hline NF-M & GAGCGCAAAGACTACCTGAAGA & CAGCGATTTCTATATCCAGAGCC \\
\hline MBP & TTAGCTGAATTCGCGTGTGG & GAGGAAGTGAATGAGCCGGTTA \\
\hline Nestin & CAGCTGGCGCACCTCAAGATG & AGGGAAGTTGGGCTCAGGACTGG \\
\hline GAD & GCGCCATATCCAACAGTGACAG & GCCAGCAGTTGCATTGACATAA \\
\hline GATA & CCAAGCAGGACTCTTGGAAC & CAGCGTGTAAAGGCATCTGA \\
\hline Nkx2.5 & TATAACGCCTACCCCGCCTAT & TATAACGCCTACCCCGCCTAT \\
\hline MLC-2A & ACAGAGTTTATGAGGTGCCCC & AAGGTGAAGTGTCCCAGAGG \\
\hline MLC-2V & TATTGGAACATGGCCTCTGGAT & GGTGCTGAAGGCTGATTACGTT \\
\hline Collagen II & GAAACCATCAATGGTGGCTTCC & CGATAACAGTCTTGCCCCACTT \\
\hline Aggrecan & GCCTTGAGCAGTTCACCTTC & CTCTTCTACGGGGACAGCAG \\
\hline COMP & CAGGACGACTTTGATGCAGA & AAGCTGGAGCTGTCCTGGTA \\
\hline VEGF & CGTGTACGTTGGTGCCCGCT & GCTGTAGGAAGCTCATCTCT \\
\hline$\beta$-actin & CGCACCACTGGCATTGTCAT & TTCTCCTTGATGTCACGCAC \\
\hline & & \\
\hline
\end{tabular}




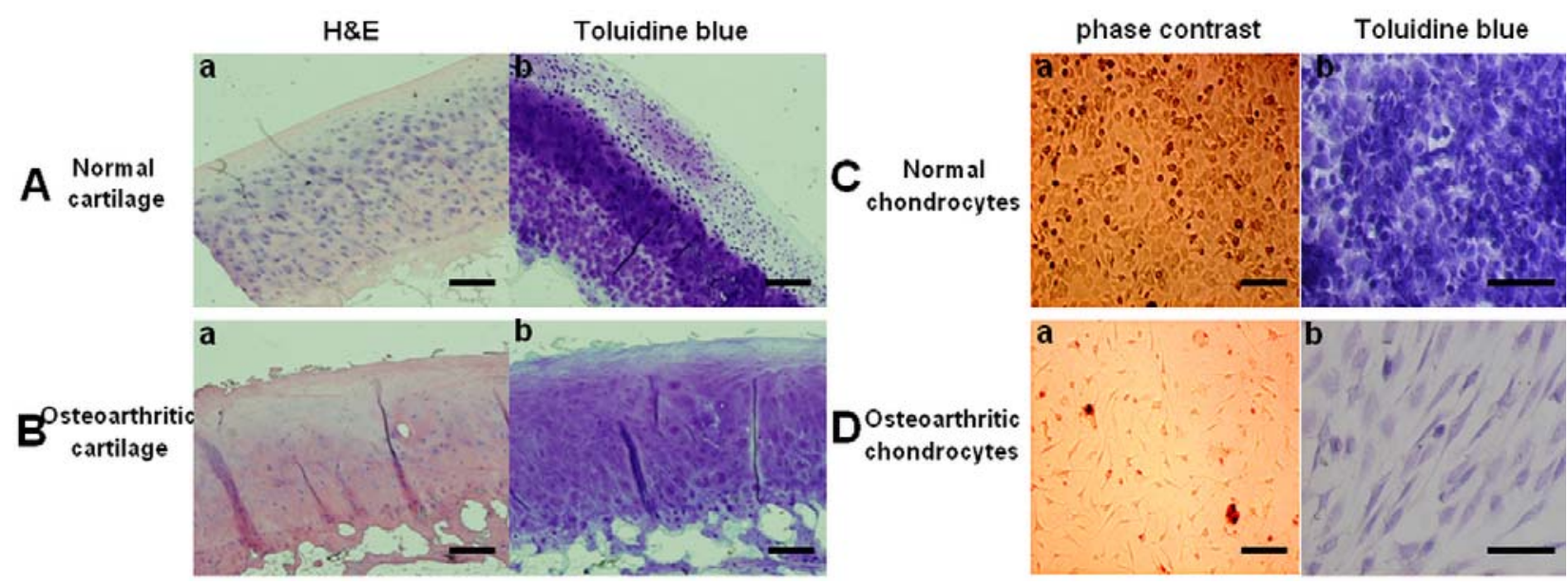

Fig. 1. Normal and osteoarthritic cartilage-derived cells. (A) Representative image of tissue from healthy AC. (B) Representative image of tissue from osteoarthritic cartilage. (C) Representative image of normal chondrocytes. (D) Representative image of OCs. Scale bars: $500 \mu \mathrm{m}(\mathbf{A}$ and $\mathbf{B}), 100 \mu \mathrm{m}(\mathbf{C}$ and $\mathbf{D})$.

A

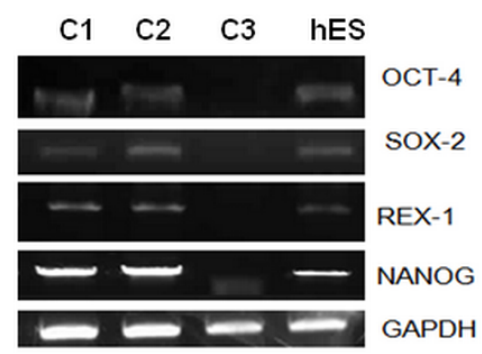

B

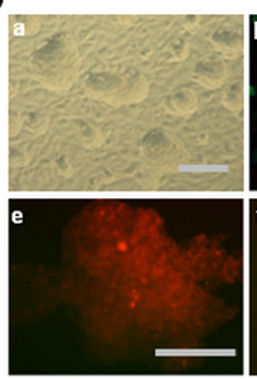

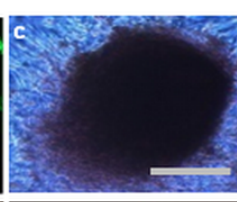

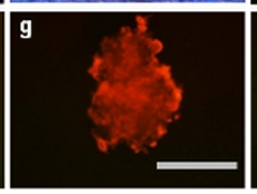

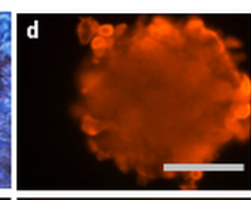

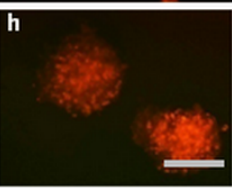

Fig. 2. Generation of iPSCs from human OCs. (A) RT-PCR revealed that human OC-derived iPSCs (C1 and C2) expressed hESC marker genes, while the other iPSCs (C3) did not. (B) The iPSC colonies were observed 2 weeks after transduction (phase contrast image (a) and fluorescence image (b), and the expression of pluripotent markers of human OC-derived iPSCs was analysed by ALP (c) and immunofluorescence staining against NANOG (d), SSEA-1 (e), SSEA-4 (f), TAR-60 (g), and TAR-81 (h). Scale bars: $200 \mu \mathrm{m}$.

to the above procedure. Then, four groups were implanted subcutaneously into the dorsal region of the mice. All animal experiments were performed in accordance with the institutional animal guidelines. Specimens from each group were harvested 6 weeks after implantation. Explants harvested from the mice were fixed, dehydrated, and embedded in paraffin. Sections were subjected to immunohistochemical analysis for collagen type II (diluted at 1:100, 53047, Abcam).

\section{Statistical analysis}

Results are reported as the mean \pm SD. Values with $P<0.05$ were considered statistically significant.

\section{Results}

\section{Histological analysis of normal and osteoarthritic cartilage-derived cells}

Healthy AC exhibited typically four intact cell layers including a superficial, transitional, deep radial, and deep calcified zone (Fig. 1Aa), and uniformly-distributed proteoglycan as the main extracellular matrix component (Fig. 1Ab). Osteoarthritic cartilage showed disappearance of cell organelles and the existence of a cell outline in some cells, and some necrotic cells (Fig. 1Ba) as well as a loss of proteoglycan (Fig. 1Bb). Chondrocytes isolated from healthy $\mathrm{AC}$ exhibited the typical round, polygonal shape (Fig. 1Ca) and had an abundance of proteoglycan, the main extracellular matrix component of chondrocytes (Fig. 1Cb). Most OCs had an elongated shape (Fig. 1Da) and exhibited a loss of proteoglycan (Fig. 1Db).

\section{Characterisation of reprogrammed human OCs}

Seven days after transduction with the four reprogramming factors, colonies with an ESC-like morphology were visible. After two weeks, colonies with an ESC-like morphology were large and round, with clear boundaries. To confirm that reprogramming of the patient OCs had occurred and that the putative iPSCs were pluripotent, we assayed the expression of undifferentiated ESC markers by RT-PCR and immunocytochemistry. RT-PCR results revealed that two of the putative human iPSCs expressed many predicted markers of undifferentiated hESCs, including OCT-4, SOX-2, REX-1 and NANOG, which were not expressed in the other putative iPSCs (Fig. 2A). Two of the three colonies exhibited strong alkaline phosphatase activity and the expressed ESC-specific surface markers including NANOG, SSEA-1, SSEA-4, TRA1-60, and 

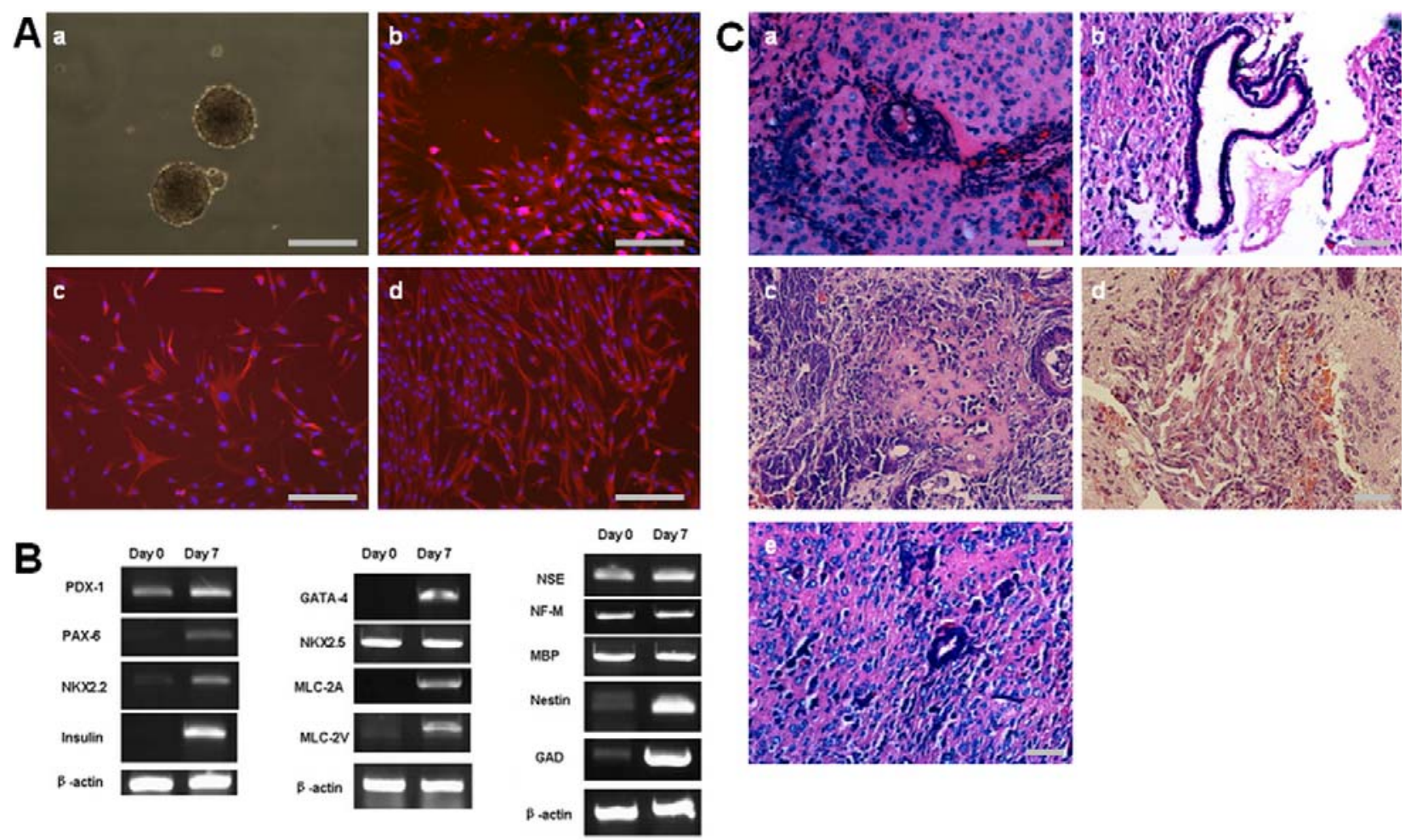

Fig. 3. Differentiation of iPSCs from human OCs in vitro and in vivo. (A) EBs were formed from human OC-derived iPSCs at day 2 (a), and differentiation markers were detected by immunofluorescent staining of AFP (b), nestin (c), and desmin (d). (B) Direct differentiation of human OC-derived iPSCs into cell lineages of all three germ layers in vitro. Differentiated cells positive for PDX-1, PAX-6, NKX2.2, and insulin (endoderm); GATA-4, NKX2.5, MLC-2A, and MLC-2V (mesoderm); NSE, NF-M, MBP, GAD, and nestin (ectoderm) as detected by RT-PCR. (C) Teratomas formed 4-6 weeks after human OC-derived iPSCs (clone 1, C1) were injected subcutaneously into four locations on nonobese diabetes/severe-combined immunodeficient (NOD/SCID) mice. Various tissues of the three germ layers were detected by H\&E staining, including gut-like epithelium (a), respiratory-like epithelium (b), bone (c), muscle (d), and neural tissue (e). Scale bars: $200 \mu \mathrm{m}$.

TRA1-81 (Fig. 2B, the image shown is of the $\mathrm{C} 1$ colony. An image of the $\mathrm{C} 2$ colony is available from the author upon request). Together, these results indicate that human iPSCs expressed pluripotent markers and shared a similar gene expression profile with hESCs.

\section{Multipotency of reprogrammed human OCs}

Next, we evaluated whether the human iPSCs could differentiate into cell types of all three germ layers in vitro through EB-mediated differentiation. The iPSCs from the osteoarthritic patient formed ball-shaped EBs with high efficiency (Fig. 3Aa) and then readily differentiated into cells with various morphologies. Immunocytochemical analyses of the EBs confirmed that human iPSCs could differentiate into cells from all three germ layers, as evidenced by the expression of $\alpha$-foetoprotein (AFP, endoderm marker), nestin (ectoderm marker), and desmin (mesoderm marker) (Fig. 3Ab,c,d).

To induce lineage-directed differentiation of human iPSCs in vitro, previously reported protocols were followed for endoderm, mesoderm and ectoderm differentiation, and the associated differentiation markers were tested by conventional RT-PCR (Miki et al., 2005) (Fig. 3B). Human iPSCs were induced to differentiate into a pancreatic (endodermal) lineage, and the differentiated cells expressed
PDX-1, PAX-6, NKX2.2, and insulin. Human iPSCs were induced to differentiate into a cardiac (mesodermal) lineage, and the differentiated cells expressed GATA-4, NKX2.5, MLC-2A, and MLC-2V. Finally, human iPSCs were induced to differentiate into a neural (ectodermal) lineage, and the differentiated cells expressed neuralrelated markers, such as NSE, NF-M, MBP, GAD, and nestin. These data demonstrate that human iPSCs can be induced directly to differentiate into cell types of all three germ layers.

Histological observation (Fig. 3C, image shown is of the $\mathrm{C} 1$ colony; an image of the $\mathrm{C} 2$ colony is available from the author upon request) showed that these teratomas possessed tissues of the three germ layers, including gutlike and respiratory-like epithelium (endoderm), bone and muscle tissues (mesoderm), and neural tissue (ectoderm).

\section{In vitro chondrogenic differentiation of reprogrammed human $\mathrm{OCs}$}

Fluorescence microscopy indicated that iPSCs appeared green at day 5 (Fig. 4Aa), while lentivirus-mediated TGF- $\beta 1$ transduced iPSCs appeared red (Fig. 4Ab) at day 5 and day 14 (Fig. 4Ac). Increased TGF- $\beta 1$ expression in the transduced iPSCs was confirmed using western blotting (Fig. 4Ad). 
A
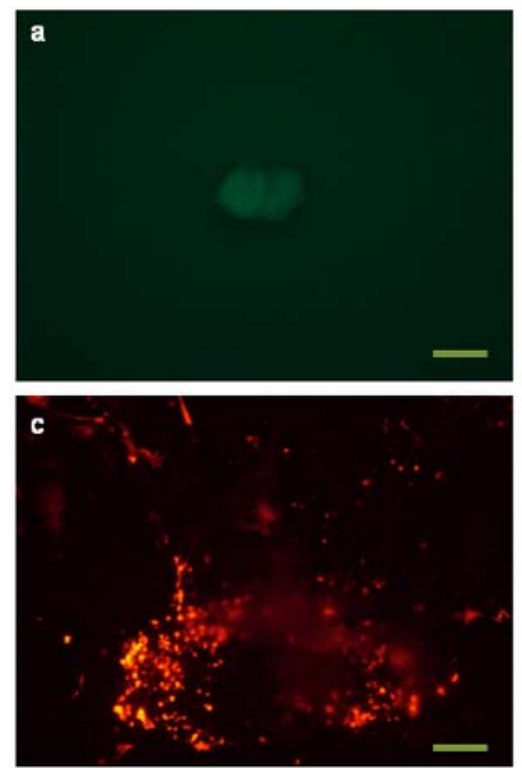

B
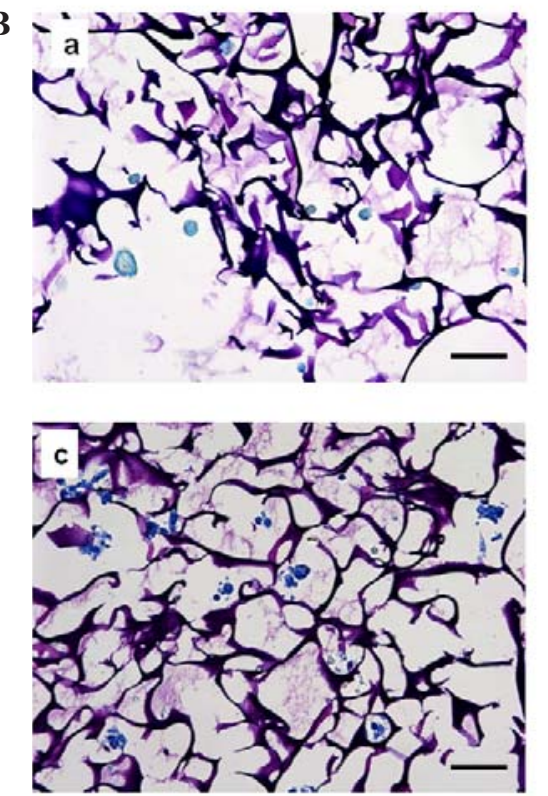

C
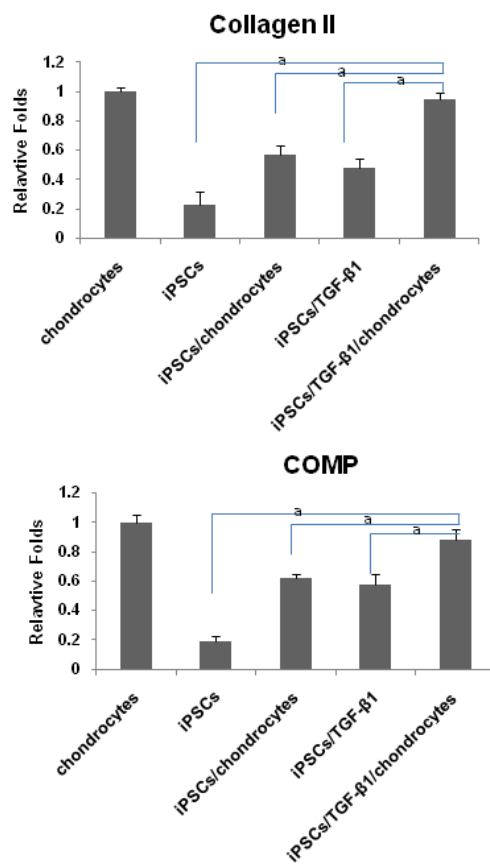

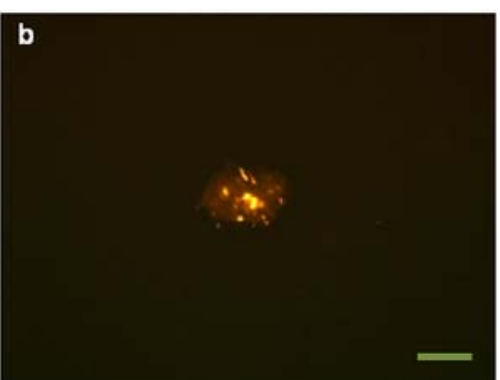

d

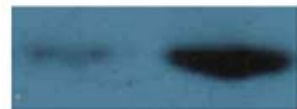

TGF- $\beta 1$

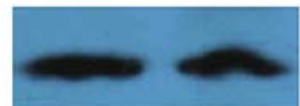

$\beta$-actin

iPSCs transfected

iPSCs
Fig. 4. In vitro chondrogenic differentiation of OC-derived iPSCs. (A) Representative images of OC-derived iPSCs with lentivirustransduced TGF- $\beta 1$ at day 5 (a and b) and 14 (c) shown by fluorescence, and a western blot against TGF- $\beta 1$ in the nontransfected and transfected iPSCs (d). (B) Toluidine blue staining of iPSCs / Alginate (a), iPSCs / Alginate / chondrocytes (b), iPSCs / TGF- $\beta 1$ / Alginate (c) and iPSCs / TGF- $\beta 1$ / Alginate / chondrocytes (d). (C) Real-time PCR results showing gene expression for the cartilage markers collagen II, aggrecan and COMP as well as the degenerative cartilage marker VEGF. ( $\left.{ }^{a}\right)$ indicates that expression levels are statistically significant different from iPSCs/ TGF- $\beta 1$ / chondrocytes in alginate matrix. Scale bars: $200 \mu \mathrm{m}$ (A), $100 \mu \mathrm{m}$ (B).
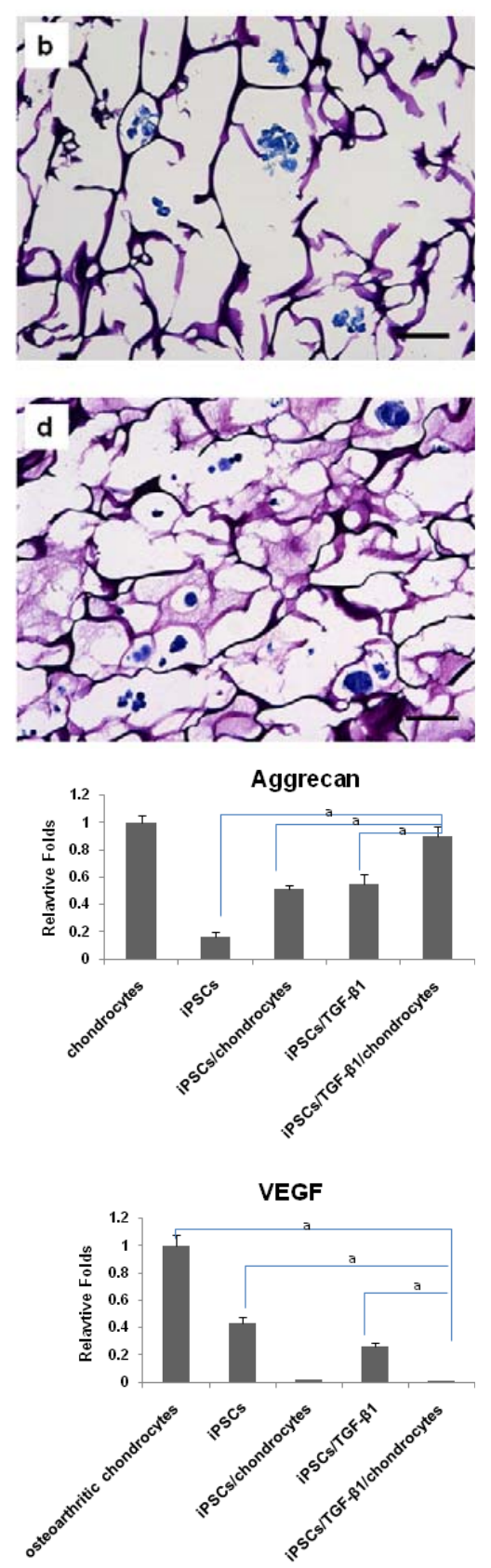

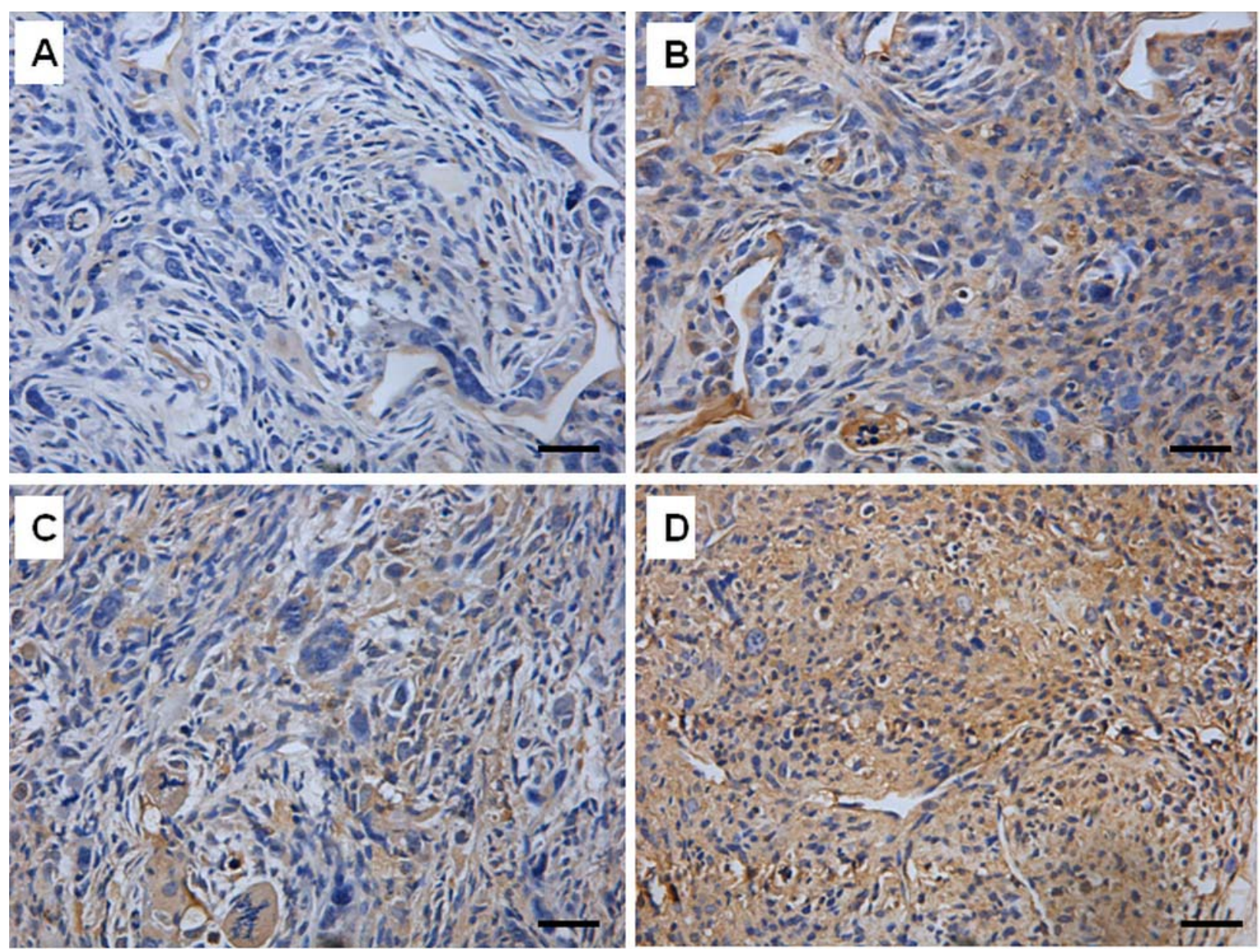

Fig. 5. Immunohistochemical analysis of ectopic cartilage formation of OC-derived iPSCs. Collagen II staining of iPSCs/Alginate (A), iPSCs/Alginate/chondrocytes (B), iPSCs/TGF- $\beta 1 /$ Alginate (C) and iPSCs /TGF- $\beta 1 /$ Alginate/ chondrocytes (D). Scale bars: $100 \mu \mathrm{m}$.

Histological analysis showed that the iPSCs/TGF- $\beta 1 /$ Alginate/chondrocytes had a round morphology, an obvious cartilage lacuna and a dense sulphated extracellular matrix that stained strongly for toluidine blue compared to the other groups (Fig. 4B).

Real-time PCR was carried out to determine the status of iPSC differentiation at transcription level. As can be seen in Fig. 4C, at day 14, the expression of the cartilage markers collagen II, aggrecan and COMP was significantly higher in iPSCs/ TGF- $\beta 1 /$ Alginate/chondrocytes than in their respective control cultures including iPSCs/Alginate, iPSCs/Alginate/chondrocytes and iPSCs/TGF- $\beta 1 /$ Alginate $(P<0.05)$, while it was less than that in the positive control group (chondrocytes) $(P>0.05)$. The expression of vascular endothelial growth factor (VEGF), a marker of degenerative chondrocytes, was high in iPSCs/ Alginate and iPSCs/TGF- $\beta 1 /$ Alginate, while it was nearly zero for iPSCs/Alginate/chondrocytes and iPSCs/TGF- $\beta 1 /$ Alginate/ chondrocytes.

In vivo chondrogenesis of reprogrammed human OCs Immunohistochemistry at 6 weeks revealed that iPSCs in the following groups including iPSCs/Alginate/ chondrocytes, iPSCs/TGF- $\beta 1$ /Alginate and iPSCs/TGF- $\beta 1$ / Alginate/chondrocytes were obviously surrounded by collagen II-positive extracellular matrix, and iPSCs stained strongly for collagen II in the iPSCs/TGF- $\beta 1 /$ Alginate/ chondrocytes group compared to the other groups (Fig. 5).

\section{Discussion}

The innate avascularity, low cellularity and low cell turnover of AC tissue represent major hurdles for natural regeneration following an injury; therefore, the development of treatments allowing successful regeneration of cartilage lesions to avoid further degeneration of focal articular defects represents a major challenge (Giovannini et al., 2010). Many studies have investigated the ability of a variety of cues to replace or repair diseased or damaged cartilage tissue (Lin et al., 2008; Goepfert et al., 2010; Ahmed et al., 2011). Recent developments in regenerative medicine have been driven by discovery of iPSCs, which based on their capacity to proliferate in an undifferentiated state, their potential to differentiate into a variety of different lineages, and their possibility of being personalised therapies, provide an intriguing alternative to the use of stem cells in tissue engineering (Kusuma and Gerecht, 2010). In this study, we have successfully reprogrammed human OCs into fully functional iPSCs by lentivirus-mediated transduction of 
the four transcription factors OCT4, SOX2, KLF4, and c-MYC. The human iPSCs from OCs displayed hESC-like morphology, expressed hESC marker genes and possessed the ability to differentiate into all three germ layers in vitro and in vivo. However, it remains unclear whether OCderived iPSCs may be used as a cell source for cartilage tissue engineering.

At present, two effective methods are used to induce the differentiation of stem cells into chondrocytes: the application of growth factors and co-culture. The traditional strategy has been to add specific growth factors directly to the media of stem cells cultured in a 3D environment. Previous research suggested that hESCderived EBs in the 3D hydrogels might differentiate toward the chondrogenic lineage in a chemically defined chondrogenic medium supplemented with TGF- $\beta 1$ (Hwang et al., 2006). Recently, hESCs cultured in medium with TGF- $\beta 1$ produced a cartilage-specific matrix and showed evidence of chondrogenic potential without EB formation (Nakagawa et al., 2009). More recently, Gong et al. (2010) demonstrated that micromass cultures of pluripotent hESCs undergo direct, rapid, progressive, and substantially uniform chondrogenic differentiation in medium containing BMP-2 plus TGF- $\beta 1$. All these findings indicate that TGF- $\beta 1$ has a significant effect on the chondrogenic differentiation of human and mouse ESCs. However, because TGF- $\beta 1$ protein is unstable due to its short half-life in vitro, gene delivery may be necessary to provide protein expression for long periods at effective concentrations (Chen et al., 2011). In our study, lentiviral-mediated TGF- $\beta 1$ was used to induce the differentiation of iPSCs into chondrocytes. RT-PCR results showed expression of cartilage-related genes, including collagen II, aggrecan, and COMP, in the TGF- $\beta 1$-transduced OC-derived iPSCs, suggesting that they acquired a chondrogenic potential. However, VEGF expression was also detected in the TGF- $\beta 1$-transduced OC-derived iPSCs, suggesting that the TGF- $\beta 1$-transduced OC-derived iPSCs have a degenerative tendency. Patient/ disease-specific iPSCs showed some of the disease features recapitulated in tissue culture after directed differentiation of iPSCs (Saha and Jaenisch, 2009; Ye et al., 2009; Liu et al., 2010), and it is possible that a similar process occurred in this study. VEGF is found at the zone of hypertrophic cartilage in the growth plate and plays an integral role in endochondral ossification and longitudinal growth of the skeleton (Gerber et al., 1999; Pufe et al., 2004). VEGF is largely downregulated in adult $\mathrm{AC}$, but this angiogenic factor is expressed by diseased chondrocytes, such as those in OA (Pufe et al., 2001). Therefore, inhibition of VEGF expression will most likely contribute to the differentiation of OC-derived iPSCs into chondrocytes.

Co-culture is the other method used for the differentiation of stem cells into chondrocytes. The primary advantage of co-culture is that the autocrine and paracrine factors secreted by one cell type readily interact with the other type of cell without direct cell-cell contact, thus mimicking, to a certain extent, the in vivo cartilage environment in which the transplanted cells are surrounded by native chondrocytes residing within the tissue (Mo et al., 2009; Aung et al., 2011). Moreover, it allows for cellular crosstalk, instruction, and stabilisation of cellular phenotype by direct physical contact between the surface receptors of different cells, which may lead to more efficient transduction of molecular signals coordinating chondrogenic differentiation (Heng et al., 2004). Co-culturing human MSCs with human ACs has been shown to enhance the mechanical properties and cartilage-specific ECM content of tissue-engineered cartilage formed from MSCs seeded in hyaluronic acid (HA) hydrogels and to decrease expression of collagen type X by MSCs, an important marker of MSC hypertrophy (Bian et al., 2011). Co-culturing equine MSCs with mature ACs improved expression of cartilage-specific genes and produced a more homogeneous production of ECM within the newly formed co-cultured cartilage (Lettry et al., 2010). Furthermore, the chondrogenic potential of hESCs could be enhanced in a high-density micromass pellet co-cultured with irradiated human chondrocytes, and co-culture resulted in a significantly decreased osteogenic potential (Bigdeli et al., 2009). In our study, differentiation of OCderived iPSCs was induced by co-culturing with ACs, as demonstrated by the expression of cartilage-specific genes. Although the level of cartilage-specific genes in the co-culture system was lower than that in the transfected OC-derived iPSCs, VEGF expression was not detected, possibly due to endogenous inhibition of angiogenesis by factors, including metastatin, present in adult cartilage tissue (Moses et al., 1999; Liu et al., 2001).

One possible approach to overcome the individual shortcoming of the gene-transfer and co-culture systems in the differentiation of OC-derived iPSCs into chondrocytes would be to mix human chondrocytes with TGF- $\beta 1$ transduced OC-derived iPSCs in a co-culture system. In our co-culture system, co-culturing the TGF- $\beta 1$ transduced OC-derived iPSCs with ACs promoted the differentiation of OC-derived iPSCs into chondrocytes by enhancing the cartilage-specific gene expression, inhibiting VEGF expression and preventing degeneration of the chondrocytes.

To further validate the chondrogenic capacity of reprogrammed human OCs, in vivo study was performed. In ectopic cartilage formation, direct evidence of chondrogenic differentiation was collected by immunohistochemical study of collagen II. Collagen II, which is characteristic of a cartilage matrix, was present at higher level in the iPSCs/TGF- $\beta 1 /$ Alginate/chondrocytes group, as compared to that in the iPSCs/Alginate/chondrocytes group and the iPSCs/TGF- $\beta 1 /$ Alginate group. Histological examination of iPSCs revealed the obvious cartilage tissue formation generated in the co-culture of TGF- $\beta 1$-transfected OCderived iPSCs with ACs in alginate matrix at 6 weeks.

\section{Conclusion}

Taken together, our data indicate that iPSCs can be generated from OCs using the defined factors and that in vitro co-culture of TGF- $\beta 1$-transfected OC-derived iPSCs with ACs in alginate matrix results in significantly improved chondrogenesis of iPSCs as shown by the gene expression profiles and the histological staining. Additionally, in vivo study also revealed the obvious cartilage tissue formed in the co-culture of TGF- $\beta 1$ - 
transfected OC-derived iPSCs with ACs in alginate matrix. This combinational strategy will promote the use of iPSCderived tissue in tissue engineering.

\section{Acknowledgements}

This work was supported by Innovation Program of Shanghai Municipal Education Commission (No. 09YZ107) and the Intramural Research Program of the National Institutes of Health, National Cancer Institute.

\section{References}

Ahmed N, Taylor DW, Wunder J, Nagy A, Gross AE, Kandel RA (2010) Passaged human chondrocytes accumulate extracellular matrix when induced by bovine chondrocytes. J Tissue Eng Regen Med 4: 233-241.

Ahmed TA, Giulivi A, Griffith M, Hincke M (2011) Fibrin glues in combination with mesenchymal stem cells to develop a tissue-engineered cartilage substitute. Tissue Eng Part A 17: 323-335.

Altman R, Alarcon G, Appelrouth D, Bloch D, Borenstein D, Brandt K, Brown C, Cooke TD, Daniel W, Gray R, et al. (1990) The American College of Rheumatology criteria for the classification and reporting of osteoarthritis of the hand. Arthritis Rheum 33: 16011610 .

Aung A, Gupta G, Majid G, Varghese S (2011) Osteoarthritic chondrocyte-secreted morphogens induce chondrogenic differentiation of human mesenchymal stem cells. Arthritis Rheum 63: 148-158.

Bian L, Zhai DY, Mauck RL, Burdick JA (2011) Coculture of human mesenchymal stem cells and articular chondrocytes reduces hypertrophy and enhances functional properties of engineered cartilage. Tissue Eng Part A 17: 1137-1145.

Bigdeli N, Karlsson C, Strehl R, Concaro S, Hyllner J, Lindahl A (2009) Coculture of human embryonic stem cells and human articular chondrocytes results in significantly altered phenotype and improved chondrogenic differentiation. Stem Cells 27: 1812-1821.

Chen J, Chen H, Li P, Diao H, Zhu S, Dong L, Wang R, Guo T, Zhao J, Zhang J (2011) Simultaneous regeneration of articular cartilage and subchondral bone in vivo using MSCs induced by a spatially controlled gene delivery system in bilayered integrated scaffolds. Biomaterials 32: 4793-4805.

Dehne T, Karlsson C, Ringe J, Sittinger M, Lindahl A (2009) Chondrogenic differentiation potential of osteoarthritic chondrocytes and their possible use in matrix-associated autologous chondrocyte transplantation. Arthritis Res Ther 11: R133.

DeLise AM, Fischer L, Tuan RS (2000) Cellular interactions and signaling in cartilage development. Osteoarthritis Cartilage 8: 309-334.

Gaspar-Maia A, Alajem A, Meshorer E, RamalhoSantos M (2011) Open chromatin in pluripotency and reprogramming. Nat Rev Mol Cell Biol 12: 36-47.
Gerber HP, Vu TH, Ryan AM, Kowalski J, Werb Z, Ferrara N (1999) VEGF couples hypertrophic cartilage remodeling, ossification and angiogenesis during endochondral bone formation. Nat Med 5: 623-628.

Giovannini S, Diaz-Romero J, Aigner T, Heini P, Mainil-Varlet P, Nesic D (2010) Micromass co-culture of human articular chondrocytes and human bone marrow mesenchymal stem cells to investigate stable neocartilage tissue formation in vitro. Eur Cell Mater 20: 245-259.

Goepfert C, Slobodianski A, Schilling AF, Adamietz P, Portner R (2010) Cartilage engineering from mesenchymal stem cells. Adv Biochem Eng Biotechnol 123: 163-200.

Gong G, Ferrari D, Dealy CN, Kosher RA (2010) Direct and progressive differentiation of human embryonic stem cells into the chondrogenic lineage. J Cell Physiol 224: 664-671.

Hattori K, Ohgushi H (2009) [Progress of research in osteoarthritis. Tissue engineering therapy for osteoarthritis]. Clin Calcium 19: 1621-1628.

Heng BC, Cao T, Lee EH (2004) Directing stem cell differentiation into the chondrogenic lineage in vitro. Stem Cells 22: 1152-1167.

Honda A, Hirose M, Hatori M, Matoba S, Miyoshi H, Inoue K, Ogura A (2010) Generation of induced pluripotent stem cells in rabbits: potential experimental models for human regenerative medicine. J Biol Chem 285: $31362-$ 31369.

Hwang NS, Kim MS, Sampattavanich S, Baek JH, Zhang Z, Elisseeff J (2006) Effects of three-dimensional culture and growth factors on the chondrogenic differentiation of murine embryonic stem cells. Stem Cells 24: 284-291.

Ingber DE, Mow VC, Butler D, Niklason L, Huard J, Mao J, Yannas I, Kaplan D, Vunjak-Novakovic G (2006) Tissue engineering and developmental biology: going biomimetic. Tissue Eng 12: 3265-3283.

Itoh M, Kiuru M, Cairo MS, Christiano AM (2011) Generation of keratinocytes from normal and recessive dystrophic epidermolysis bullosa-induced pluripotent stem cells. Proc Natl Acad Sci U S A 108: 8797-8802.

Koelling S, Kruegel J, Irmer M, Path JR, Sadowski B, Miro X, Miosge N (2009) Migratory chondrogenic progenitor cells from repair tissue during the later stages of human osteoarthritis. Cell Stem Cell 4: 324-335.

Kusuma S, Gerecht S (2010) Engineering blood vessels using stem cells: innovative approaches to treat vascular disorders. Expert Rev Cardiovasc Ther 8: 1433-1445.

Lettry V, Hosoya K, Takagi S, Okumura M (2010) Coculture of equine mesenchymal stem cells and mature equine articular chondrocytes results in improved chondrogenic differentiation of the stem cells. Jpn J Vet Res 58: 5-15.

Li W, Zhou H, Abujarour R, Zhu S, Young Joo J, Lin T, Hao E, Scholer HR, Hayek A, Ding S (2009) Generation of human-induced pluripotent stem cells in the absence of exogenous Sox2. Stem Cells 27: 2992-3000.

Lin L, Zhou C, Wei X, Hou Y, Zhao L, Fu X, Zhang J, Yu C (2008) Articular cartilage repair using dedifferentiated articular chondrocytes and bone morphogenetic protein 4 in a rabbit model of articular cartilage defects. Arthritis Rheum 58: 1067-1075. 
Liu H, Ye Z, Kim Y, Sharkis S, Jang YY (2010) Generation of endoderm-derived human induced pluripotent stem cells from primary hepatocytes. Hepatology 51: 1810-1819.

Liu N, Lapcevich RK, Underhill CB, Han Z, Gao F, Swartz G, Plum SM, Zhang L, Green SJ (2001) Metastatin: a hyaluronan-binding complex from cartilage that inhibits tumor growth. Cancer Res 61: 1022-1028.

Lohmander LS, Roos EM (2007) Clinical update: treating osteoarthritis. Lancet 370: 2082-2084.

Merceron C, Portron S, Masson M, Lesoeur J, Fellah BH, Gauthier O, Geffroy O, Weiss P, Guicheux J, Vinatier C (2011) The effect of two and three dimensional cell culture on the chondrogenic potential of human adipose-derived mesenchymal stem cells after subcutaneous transplantation with an injectable hydrogel. Cell Transplant, in press.

Miki T, Lehmann T, Cai H, Stolz DB, Strom SC (2005) Stem cell characteristics of amniotic epithelial cells. Stem Cells 23: 1549-1559.

Mo XT, Guo SC, Xie HQ, Deng L, Zhi W, Xiang Z, Li XQ, Yang ZM (2009) Variations in the ratios of co-cultured mesenchymal stem cells and chondrocytes regulate the expression of cartilaginous and osseous phenotype in alginate constructs. Bone 45: 42-51.

Moses MA, Wiederschain D, Wu I, Fernandez CA, Ghazizadeh V, Lane WS, Flynn E, Sytkowski A, Tao T, Langer R (1999) Troponin I is present in human cartilage and inhibits angiogenesis. Proc Natl Acad Sci U S A 96: 2645-2650.

Nakagawa T, Lee SY, Reddi AH (2009) Induction of chondrogenesis from human embryonic stem cells without embryoid body formation by bone morphogenetic protein 7 and transforming growth factor beta1. Arthritis Rheum 60: $3686-3692$.

Pufe T, Petersen W, Tillmann B, Mentlein R (2001) The splice variants VEGF121 and VEGF189 of the angiogenic peptide vascular endothelial growth factor are expressed in osteoarthritic cartilage. Arthritis Rheum 44: 1082-1088.

Pufe T, Petersen WJ, Miosge N, Goldring MB, Mentlein R, Varoga DJ, Tillmann BN (2004) Endostatin/collagen XVIII - an inhibitor of angiogenesis - is expressed in cartilage and fibrocartilage. Matrix Biol 23: 267-276.

Reginster JY (2002) The prevalence and burden of arthritis. Rheumatology (Oxford) 41 Supp 1: 3-6.

Saha K, Jaenisch R (2009) Technical challenges in using human induced pluripotent stem cells to model disease. Cell Stem Cell 5: 584-595.

Takahashi K, Tanabe K, Ohnuki M, Narita M, Ichisaka T, Tomoda K, Yamanaka S (2007) Induction of pluripotent stem cells from adult human fibroblasts by defined factors. Cell 131: 861-872.

Takahashi K, Yamanaka S (2006) Induction of pluripotent stem cells from mouse embryonic and adult fibroblast cultures by defined factors. Cell 126: 663-676.

Tuan RS (2004) Biology of developmental and regenerative skeletogenesis. Clin Orthop Relat Res 427: S105-117.

Woolf AD, Pfleger B (2003) Burden of major musculoskeletal conditions. Bull World Health Organ 81: 646-656.
Ye Z, Zhan H, Mali P, Dowey S, Williams DM, Jang YY, Dang CV, Spivak JL, Moliterno AR, Cheng L (2009) Human-induced pluripotent stem cells from blood cells of healthy donors and patients with acquired blood disorders. Blood 114: 5473-5480.

Yoshida Y, Yamanaka S (2010) Recent stem cell advances: induced pluripotent stem cells for disease modeling and stem cell-based regeneration. Circulation 122: $80-87$.

\section{Discussion with Reviewers}

Reviewer I: Please discuss whether the efficiency of iPS cell generation would be the same from osteoarthritis chondrocytes and normal chondrocytes.

Authors: Dey and Evans (2011, additional reference) demonstrated that before starting any reprogramming, information about the plasticity of the starting cell was helpful as cellular reprogramming is interplay between plasticity and environmental factors like epigenetic modifications. Therefore, the efficiency of cell reprogramming varies among different cell types. The susceptibility of a somatic cell to reprogramming possibly rely its resemblance to an ES cell and/or its endogenous expression of the reprogramming genes (Gadue and Costarelis, 2008, additional reference). Aasen et al. (2008, additional reference) showed that human keratinocytes, which express much higher levels of the reprogramming factors KLF4 and c-MYC, were more efficient than fibroblasts in iPSCs generation. Genetic memory can also influence the ability of iPS cells to be reprogrammed. Alonge et al. (2004, additional reference) suggested that the expression of c-MYC cannot be found in the chondrocytes of normal articular cartilage but its expression existed in the chondrocytes in the entire cartilage mantle of soteophytes by the histological study. Pelletier et al. (1993, additional reference) demonstrated that in OA cartilage, chondrocytes at the superficial and middle layers as well as in fibrillated areas were found to be involved in the synthesis of c-MYC protein. Therefore, we concluded that the efficiency of iPSCs generation was higher in osteoarthritic chondrocytes than that in normal chondrocytes because of the high expression of endogenous c-MYC in osteoarthritic chondrocytes.

Reviewer II: I wonder if the iPS cells can still maintain their phenotype after TGF- $\beta 1$ transfection. Please comment.

Authors: In our study, the iPSCs did not maintain their phenotype after TGF- $\beta 1$ transfection and the transfected iPSCs differentiated towards chondrogenic phenotype. TGF- $\beta$ is known to be a potent inducer of stem cell chondrogenic differentiation. Besides some reports mentioned in our manuscript, other researches also suggested that ESCs might differentiate toward the chondrogenic lineage in a chemically defined chondrogenic medium in the presence of TGF- $\beta 1$ (Hwang et al., 2008; Toh et al., 2009, additional references). Additionally, transforming growth factor- $\beta$ has also been 
shown to be involved in maintaining the pluripotency of ESCs). Recently, Li et al. (2010, additional reference) demonstrated that MSCs (mesenchymal stem cells)-like cells can be generated from fibroblast-derived iPSCs by brief exposure to TGF- $\beta 1$ in the presence of retinoic acid. There was some difference between Li et al. report and our experiment. In our experiment, iPSCs were stably transfected with TGF- $\beta 1$ but in the Li et al. study, iPSCs were treated by brief exposure to TGF- $\beta 1$. In addition, epigenetic memory of the somatic cell of origin is retained in the iPSCs (Chun et al., 2010, additional reference) and that the memory may influence their directed differentiation potential after treated with TGF- $\beta 1$. Our iPSC cells were acquired from osteoarthritic chondrocytes and other iPSCs were mainly generated from fibroblasts.

Reviewer II: What is the clinical relevance or any potential application, risk and cost issues? These need to be discussed clearly.

Authors: iPSCs were generated by introducing the reprogramming factors, such as OCT4, SOX2, KLF4 and c-MYC, into human somatic cells. The iPSC technology offers great clinical potential in regenerative medicine as well as in disease modelling and drug discovery. Moreover, iPSCs also overcome the problem of immune rejection as well as social and religious concerns. Although in last few years iPSCs have been generated in different systems, generating and extensively characterising the clinical grade iPSCs for cell therapy in humans is still in its infancy (Kiskinis and Eggan, 2010, additional reference). Recently, several groups have successfully generated patient-specific iPSCs from donors suffering of different disorders and differentiated these cells into cells that have a crucial role in the disease (Dimos et al., 2008; Ebert et al., 2009, additional references). Human patient iPSC based models could not only be used to study the dynamics of diseases, but also be employed in screening of new drugs. Every year billions of dollars are used in drug discovery, employing animal modes. Animal models had been crucial in the investigation of disease mechanisms, but fundamental developmental, biochemical, and physiological differences exist between mice and humans, which leads to the large numbers of failed clinical trials (Kiskinis and Eggan, 2010, additional reference). iPSC technology offers the way to obtain drug efficacy and toxicity data in a diseaserelevant manner, not only at the earliest stages of drug development but also throughout the drug discovery process that guarantees lower attrition at the expensive, late stages of the clinical pipeline (Grskovic et al., 2011, additional reference). Therefore, human patient iPSC based models will limit the need for animal studies, increase the safety and efficacy of known drugs or potential drug candidates and considerably reduce the time and money required to develop new drugs. However, the transgene integration sites and alteration of the endogenous genomic organization may cause a negative safety issue while considering medical applications (Okita and Yamanaka, 2011, additional reference). Therefore, a comprehensive knowledge of the reprogramming process would be crucial for future clinical applications of iPSCs.

\section{Additional References}

Aasen T, Raya A, Barrero MJ, Garreta E, Consiglio A, Gonzalez F, Vassena R, Bilic J, Pekarik V, Tiscornia G, Edel M, Boue S, Izpisua Belmonte JC (2008) Efficient and rapid generation of induced pluripotent stem cells from human keratinocytes. Nat Biotechnol 26: 1276-1284.

Alonge TO, Rooney P, Oni OO (2004) Oncogene expression in the peri-articular osteophytes. West Afr J Med 23: 187-190.

Chun YS, Chaudhari P, Jang YY (2010) Applications of patient-specific induced pluripotent stem cells; focused on disease modeling, drug screening and therapeutic potentials for liver disease. Int J Biol Sci 6: 796-805.

Dey D, Evans GR (2011) Generation of induced pluripotent stem (iPS) cells by nuclear reprogramming. Stem Cells Int 2011: 619583.

Dimos JT, Rodolfa KT, Niakan KK, Weisenthal LM, Mitsumoto H, Chung W, Croft GF, Saphier G, Leibel R, Goland R, Wichterle H, Henderson CE, Eggan K (2008) Induced pluripotent stem cells generated from patients with ALS can be differentiated into motor neurons. Science 321: 1218-1221.

Ebert AD, Yu J, Rose FF, Jr., Mattis VB, Lorson CL, Thomson JA, Svendsen CN (2009) Induced pluripotent stem cells from a spinal muscular atrophy patient. Nature 457: 277-280.

Gadue P, Cotsarelis G (2008) Epidermal cells rev up reprogramming. Nat Biotechnol 26: 1243-1244.

Grskovic M, Javaherian A, Strulovici B, Daley GQ (2011) Induced pluripotent stem cells - opportunities for disease modelling and drug discovery. Nat Rev Drug Discov 10: 915-929.

Hwang NS, Varghese S, Elisseeff J (2008) Derivation of chondrogenically-committed cells from human embryonic cells for cartilage tissue regeneration. PLoS One 3: e2498.

Kiskinis E, Eggan K (2010) Progress toward the clinical application of patient-specific pluripotent stem cells. J Clin Invest 120: 51-59.

Li F, Bronson S, Niyibizi C (2010) Derivation of murine induced pluripotent stem cells (iPS) and assessment of their differentiation toward osteogenic lineage. J Cell Biochem 109: 643-652.

Okita K, Yamanaka S (2011) Induced pluripotent stem cells: opportunities and challenges. Philos Trans R Soc Lond B Biol Sci 366: 2198-2207.

Pelletier JP, Faure MP, DiBattista JA, Wilhelm S, Visco D, Martel-Pelletier J (1993) Coordinate synthesis of stromelysin, interleukin-1, and oncogene proteins in experimental osteoarthritis. An immunohistochemical study. Am J Pathol 142: 95-105.

Toh WS, Guo XM, Choo AB, Lu K, Lee EH, Cao $\mathrm{T}$ (2009) Differentiation and enrichment of expandable chondrogenic cells from human embryonic stem cells in vitro. J Cell Mol Med 13: 3570-3590. 